\title{
Left ventricular assist device failure due to outflow graft compression by thrombofibrotic exudate
}

\author{
Gregory R. Jackson, MD, ${ }^{a}$ Timothy Brand, MD, ${ }^{b}$ Jason N. Katz, MD, MHS, ${ }^{\mathrm{c}}$ and \\ John S. Ikonomidis, MD, PhD, ${ }^{\text {C }}$ Chapel Hill, NC
}

\footnotetext{
From the ${ }^{\mathrm{a}}$ Divisions of Cardiology, UNC Center for Heart and Vascular Care, ${ }^{\mathrm{b}}$ Cardiothoracic Surgery, and ${ }^{\mathrm{c}}$ Cardiology and Pulmonary/Critical Care Medicine, University of North Carolina at Chapel Hill, Chapel Hill, NC

Disclosures: Authors have nothing to disclose with regard to commercial support.

Received for publication July 6, 2018; revisions received Sept 26, 2018; accepted for publication Oct 17, 2018; available ahead of print Nov 30, 2018

Address for reprints: John S. Ikonomidis, MD, PhD, Division of Cardiothoracic Surgery, University of North Carolina at Chapel Hill, 3034 Burnett Womack Building, 160 Dental Cir, Chapel Hill, NC 27599-7065 (E-mail: john_ikonomidis@med.unc.edu).

J Thorac Cardiovasc Surg 2019;157:e259-61

$0022-5223 / \$ 36.00$

Copyright (C) 2018 by The American Association for Thoracic Surgery

https://doi.org/10.1016/j.jtcvs.2018.10.069
}

The implantation of left ventricular assist devices (LVADs) is a mainstay of the surgical therapy for patients with heart failure. In an effort to reduce the potential morbidity associated with repeat operations for either LVAD exchanges or transplants, many surgeons use additional materials to cover parts of the device, either to prevent adverse entry (ie, outflow graft) or to facilitate ease of removal (ie, device body). ${ }^{1}$ We present 3 cases of LVAD failure due to extrinsic outflow graft compression with thrombofibrotic exudate caused by use of an external outflow graft protective wrap. An institutional review board waiver was obtained for the preparation of this report.

The first patient, a 68-year-old woman with history of rheumatoid arthritis, breast cancer treated with radical mastectomy, chemotherapy-induced chronic systolic heart failure (ejection fraction 25\%), and a HeartMate II (St Jude Medical, St Paul, Minn) LVAD presented to the hospital with unrelenting low-flow alarms on her LVAD controller, cough, and shortness of breath 26 months after LVAD implantation. Upon admission to the hospital, she underwent transthoracic echocardiography with speed modification at LVAD speeds increasing from 9000 revolutions per minute (RPM) to 10,200 RPM in increments of 200 RPM. Echocardiography showed no change in left ventricular internal diastolic diameter $(5.5-5.6 \mathrm{~cm})$ despite increasing speeds, and no changes in LVAD parameters (ie, power, flow, or pulsatility index) at all speeds throughout the study. These findings raised concern for LVAD device malfunction. Cardiac computed tomography with angiography (CCTA) demonstrated a near-vertical orientation of the LVAD inflow cannula at the left ventricular apex with an unobstructed contrast column. There was severe stenosis at the distal aspect of the outflow graft near the anastomosis with the ascending aorta, most likely due to thrombus. The patient underwent re-do sternotomy with revision of

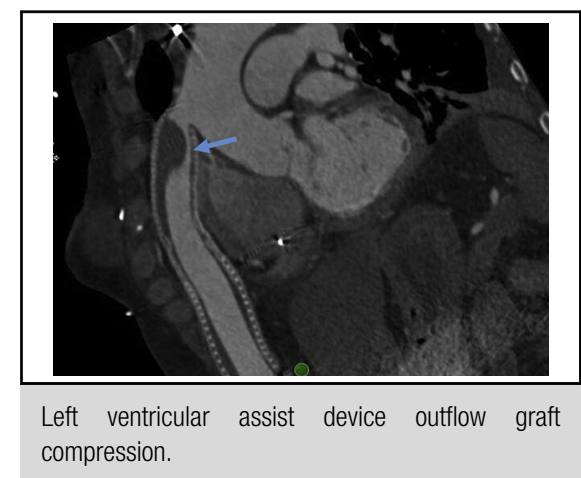

Central Message

Three cases of LVAD outflow graft compression by material accumulated within a polytetrafluoroethylene external protective graft are presented.

See Commentary on page e263.

the HeartMate II outflow graft. Intraoperative surgical findings revealed visible severe external compression of the graft originating between the graft and a surrounding Gore-Tex (W.L. Gore and Associates, Newark, Del) wrap used for reinforcement during initial device implantation. With the patient on cardiopulmonary bypass, the outflow graft was clamped and the LVAD was turned off. The distal portion of the outflow graft at the anastomosis was transected. The proximal portion of the outflow graft was disconnected from the pump body and inspected. There was no evidence of thrombus within the graft and no evidence of pseudoaneurysm at the graft-aorta anastomosis site. A new outflow graft was placed with anastomosis to the ascending aorta. The pump was restarted and flow rates optimized while in the operating room. The patient was successfully extubated and weaned from inotropic support on postoperative day 0 . There were no pulsatility index events or low-flow alarms after surgery, and after an uncomplicated recovery she was discharged to home. She has had no further LVAD alarms and no symptoms of suboptimal LVAD unloading during the 7 months since her operation.

A second patient, a 46-year-old man with nonischemic cardiomyopathy on 4.5 years of support (HeartWare Assist Device; Medtronic, Minneapolis, Minn) presented with progressive heart failure symptoms and accompanied lowflow alarms and increased power irregularities on device 

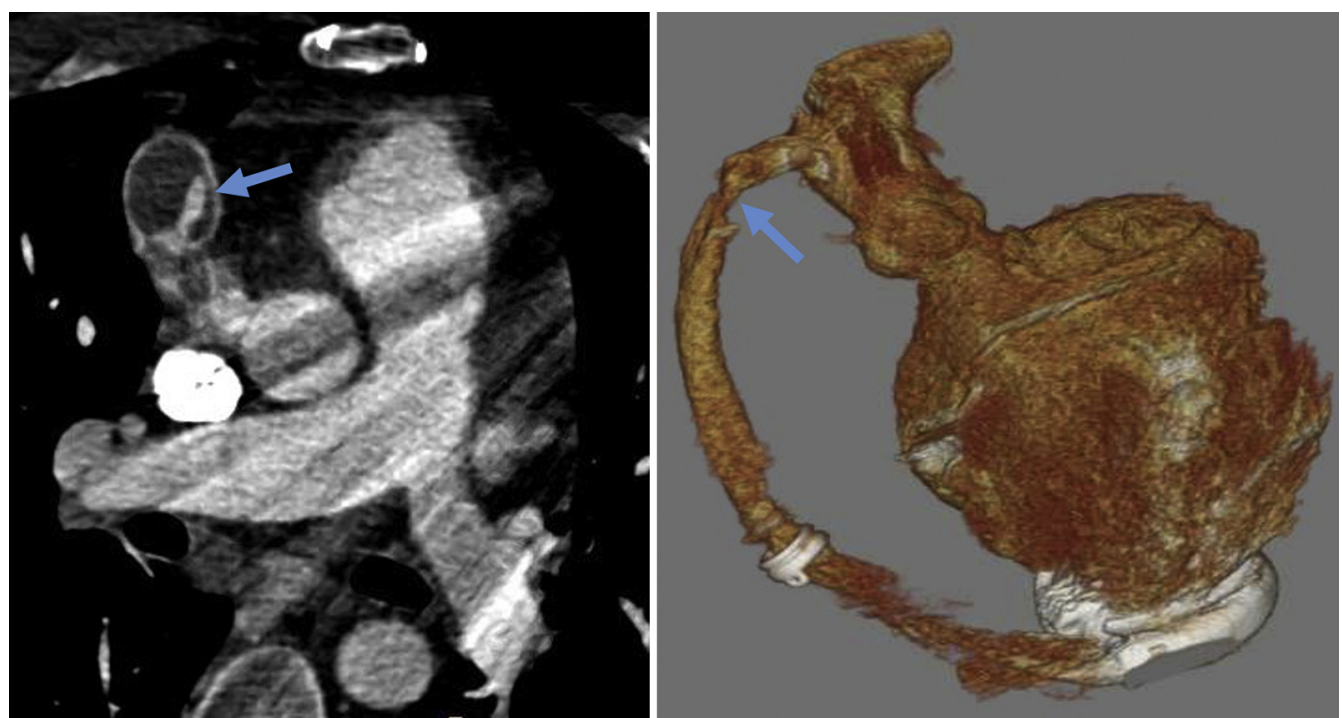

FIGURE 1. Cardiac computed tomography scan with angiography demonstrating severe stenosis at the distal aspect of the left ventricular assist device outflow graft near the anastomoses with the ascending aorta (blue arrow) in Patient 2. Left, Short axis view. Right, 3-Dimensional reconstruction.

interrogation. Transthoracic echocardiogram demonstrated severely dilated left ventricle (left ventricular internal diastolic diameter, $9.2 \mathrm{~cm}$ ) at speed of $3200 \mathrm{RPM}$, with preserved aortic valve excursion. CCTA revealed severe narrowing and obstruction of the outflow graft (Figure 1) suggestive of external compression. The patient underwent surgical intervention with removal of the anterior portion of the Gore-Tex wrap and evacuation of exudate from around the outflow graft, resulting in immediate resolution of low flow. The patient had reduced diuretic requirements with adequate pump support and improvement in functional status over the following weeks.
A third patient, a 29-year-old man with history of nonischemic cardiomyopathy on LVAD support for 3.5 years (HeartMate II) presented with several months of progressive heart failure symptoms requiring repeat hospitalization for intravenous diuretics and temporary inotropic support. Several weeks after hospital discharge, he was readmitted with Torsades de pointes requiring cardioversion, severe biventricular failure, and cardiogenic shock requiring inotropic support and intravenous diuretics. He did not have any low-flow alarms preceding these events. CCTA demonstrated significant thrombotic material throughout the outflow graft, with marked narrowing of contrast flow
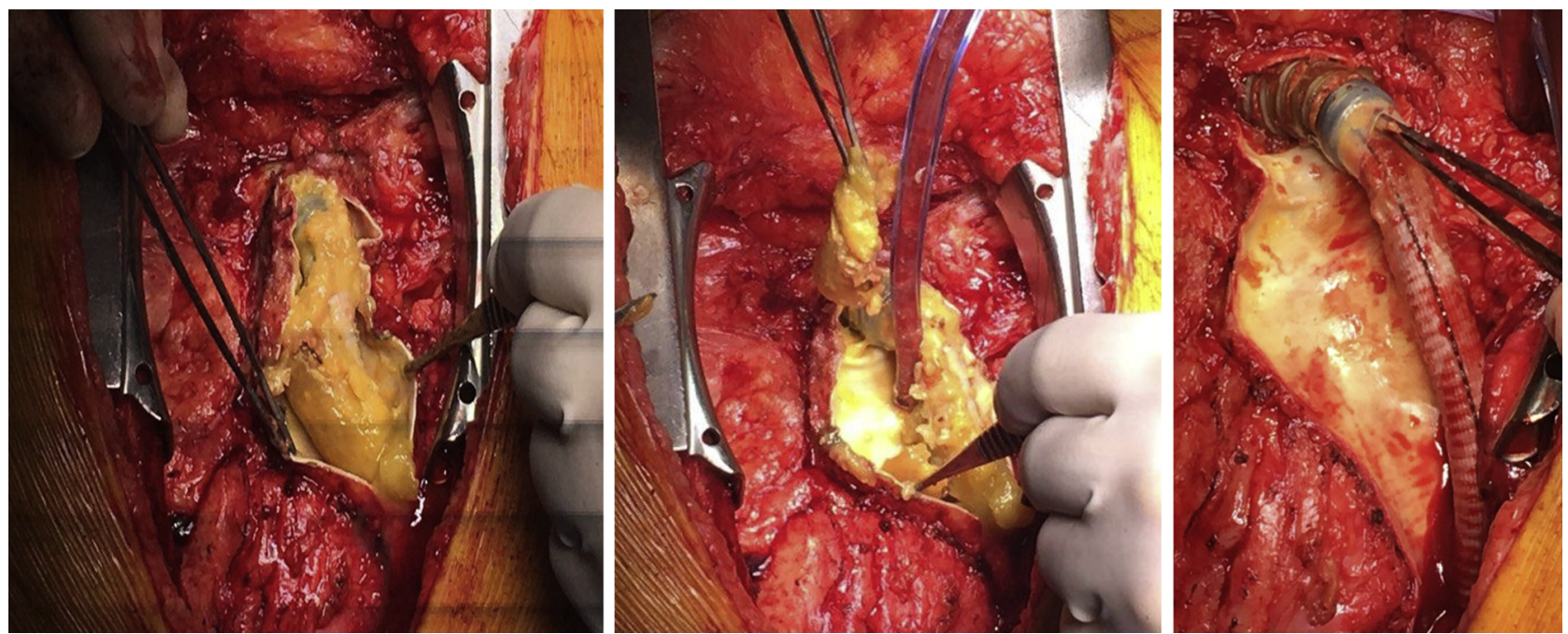

FIGURE 2. Cardiac computed tomography scan with angiography demonstrating significant thrombofibrotic material throughout the outflow graft with marked narrowing of contrast flow in the more proximal aspect in Patient 3. Left, Short axis view. Right, Long axis view. 
in the more proximal aspect. The patient was taken to the operating room to unroof and relieve this obstruction (Figure 2). He recovered postoperatively with improvement in symptoms and decreased pharmacologic support.

\section{DISCUSSION}

The above cases present a cause of LVAD pump failure secondary to external compression and obstruction of the outflow graft by thrombofibrotic exudate. The surgical technique of wrapping the outflow graft has been employed to reduce adhesions during subsequent heart surgeries or transplant. It is postulated that plasma and serum contents leak through the outflow graft material into the space between the outflow graft and Gore-Tex protective wrap that accumulates over time to form a collection of thrombofibrinous material. The trapping of this material results in external compression and obstruction of the outflow graft with subsequent device malfunction. In our patients, there were no unusual circumstances noted with regard to anticoagulation. Few cases of this complication have been cited in the literature, with treatments ranging from stent placement to surgical interventions. ${ }^{2}$ This complication may be avoided with placement of a less-constricting or fenestrated protective wrap, or more lateral placement of the outflow graft with adjusted surgical configuration and no protective wrap placed. Another consideration, applicable to HeartMate III and HeartWare devices, would be to leave a 2- to $3-\mathrm{cm}$ shelf of pericardium (augmented with Gore-Tex or other material) attached to the diaphragm when opening the pericardial sac. After device implantation, the outflow graft can be positioned under this shelf for additional protection as it crosses the midline.

\section{References}

1. Leprince P, Rahmati M, Bonnet N, Bors V, Rama A, Léger P, et al. Expanded polytetrafluoroethylene membranes to wrap surfaces of circulatory support devices in patients undergoing bridge to heart transplantation. Eur J Cardiothorac Surg. 2001;19:302-6.

2. Trankle CR, Quader MA, Grizzard JD, Tang DG, Shah KB, Paris K, et al. Internal versus external compression of a left ventricular assist device outflow graft: diagnosis with intravascular ultrasound and treatment with stenting. Circ Heart Fail. 2018;11:e004959. 